Homology, Homotopy and Applications, wol. 15(四), 2013, pp.267-280

\title{
ON KIRCHHOFF'S THEOREMS WITH COEFFICIENTS IN A LINE BUNDLE
}

\author{
MICHAEL J. CATANZARO, VLADIMIR Y. CHERNYAK AND \\ JOHN R. KLEIN
}

(communicated by Robert Ghrist)

\begin{abstract}
We prove 'twisted' versions of Kirchhoff's network theorem and Kirchhoff's matrix-tree theorem on connected finite graphs. Twisting here refers to chains with coefficients in a flat unitary line bundle.
\end{abstract}

\section{Introduction}

It is well-known that the classical result of Kirchhoff on the flow of electricity through a finite network admits an elegant formulation using algebraic topology $\mathbb{E}$, $\mathbb{[ N S}$, [R]. For a finite connected 1-dimensional CW complex $\Gamma$, the real cellular chain complex $\partial: C_{1}(\Gamma ; \mathbb{R}) \rightarrow C_{0}(\Gamma ; \mathbb{R})$ is a homomorphism of finite dimensional real inner product spaces with orthonormal basis given by the set of cells. When the branches of the network have unit resistance, Kirchhoff's network theorem is reflected in the statement that the restricted homomorphism

$$
\partial: B^{1}(\Gamma ; \mathbb{R}) \rightarrow B_{0}(\Gamma ; \mathbb{R})
$$

is an isomorphism, where $B_{0}(\Gamma ; \mathbb{R})$ is the vector subspace of zero-boundaries and $B^{1}(\Gamma ; \mathbb{R})$ is the orthogonal complement to the space of 1 -cycles $Z_{1}(\Gamma ; \mathbb{R}) \subset C_{1}(\Gamma ; \mathbb{R})$ (when the network has branches of varying resistance, one rescales the inner product on $C_{1}(\Gamma ; \mathbb{R})$ accordingly). Actually Kirchhoff's network theorem does more in that it provides a concrete expression for the inverse to the isomorphism (四) in terms of the set of spanning trees of $\Gamma$. The expression amounts to an explicit formula for the orthonormal projection of $C_{1}(\Gamma ; \mathbb{R})$ onto $Z_{1}(\Gamma ; \mathbb{R})$ in terms of the set of spanning trees of $\Gamma$.

A companion result of Kirchhoff, which has gotten more press, is the matrix-tree theorem, which computes the determinant of the restricted combinatorial Laplacian

$$
\partial \partial^{*}: B_{0}(\Gamma ; \mathbb{R}) \rightarrow B_{0}(\Gamma ; \mathbb{R}) .
$$

Here, $\partial^{*}$ denotes the formal adjoint to the boundary operator (for a slightly different formulation, see $[\mathbb{B}]$, p. 57]). In the unit resistance case, the result says that $\operatorname{det}\left(\partial \partial^{*}\right)$ equals the product of the number of vertices with the number of spanning trees

Received August 1, 2012, revised June 10, 2013; published on December 1, 2013.

2010 Mathematics Subject Classification: Primary: 55J15, 57M15, 57Q10 Secondary: 05C05, 05C21, $05 \mathrm{E} 45,82 \mathrm{C} 31$

Key words and phrases: graph, homology, line bundle, Kirchhoff, matrix-tree.

Article available at http://intlpress.com/HHA/v15/n2/a16 and doi:10.4310/HHA.2013.v15.n2 .a16

Copyright (C) 2013, International Press. Permission to copy for private use granted. 
of $\Gamma$. In [CCK], using ideas from statistical mechanics, we showed how the matrixtree theorem can be derived from the general case of the network theorem. We also generalized both of these results to higher dimensional CW complexes.

The main purpose of the current paper is to derive a twisted version of Kirchhoff's theorems. Here the twisting is given by taking coefficients in a complex line bundle. In physical terms, the twisted version of the network theorem turns out to model the flow of current through an electrical network in the presence of fluctuations. These fluctuations allow one to compute not only the distribution function of currents but also the generating function, via the Fourier transform.

Remark 1.1. The physics papers [CKST] and [CKS2] study the distribution of currents (i.e., homology classes in degree one) on graphs using a non-equilibrium statistical mechanics formalism. The main invariant appearing in these papers is given by averaging currents over stochastic trajectories in a certain long time and low temperature limit. From the physics point of view, one is interested in computing the distribution function. However, it is more convenient to compute the generating function, associated with the probability distribution, which are related via a Fourier transform. The latter can be done by twisting the graph Laplacian by a line bundle. It is in this sense that the study of fluctuations corresponds to twisting the Laplacian by a line bundle.

Our main result is a twisted version of Kirchhoff's projection formula (Theorem $\mathbb{\Delta}$ ). As an application, we will deduce a twisted version of the matrix-tree theorem (Theorem $\mathbb{Q}$ ). Suitably reformulated, our twisted matrix-tree theorem is actually a result of Forman $[\boldsymbol{\Psi}$, eq. (1)], which we first learned about in a recent paper of Kenyon $[\mathbf{K} \boldsymbol{\theta}$, theorem 5]. Forman's proof is combinatorial, using an explicit expression for the determinant in terms of symmetric groups. Kenyon's proof relies on the Cauchy-Binet theorem. By contrast, our approach is inspired by statistical mechanical ideas and closely follows the untwisted version appearing in [CCK].

\section{Graphs}

A graph $\Gamma$ is a $\mathrm{CW}$ complex of dimension one. We let $\Gamma_{0}$ denote the set of 0 -cells and $\Gamma_{1}$ the set of 1-cells. A 0 -cell is called a vertex and a 1-cell is called an edge. The entire structure of $\Gamma$ is given by a function

$$
\left(d_{0}, d_{1}\right): \Gamma_{1} \rightarrow \Gamma_{0} \times \Gamma_{0},
$$

which sends an edge $b$ to its initial and terminal endpoints (where the edge $b$ is oriented using its characteristic map $\chi_{b}:[0,1] \rightarrow \Gamma$ ). Given the function $(\mathbb{\Xi})$, one can reconstruct $\Gamma$ by taking

$$
\Gamma_{0} \cup\left(\Gamma_{1} \times[0,1]\right),
$$

where the union is amalgamated over the map $\Gamma_{1} \times\{0,1\} \rightarrow \Gamma_{0}$ given by $(b, 0) \mapsto$ $d_{0}(b)$ and $(b, 1) \mapsto d_{1}(b)$.

A loop edge is an edge such that $d_{0}(b)=d_{1}(b)$. If $b$ is not a loop edge, it is said to be regular.

\section{Flat line bundles on graphs}

A flat complex vector bundle $\rho$ on a graph $\Gamma$ is a rule that assigns to each vertex $i \in$ $\Gamma_{0}$ a finite rank complex vector space $V_{i}$ over $\mathbb{C}$, and to each edge $b$ with $\left(d_{0} b, d_{1} b\right)=$ 
$(i, j)$ an isomorphism

$$
\rho_{b}: V_{i} \rightarrow V_{j}
$$

We say that $\rho$ is unitary if each $V_{i}$ is a hermitian inner product space and each $\rho_{b}$ is unitary. In this paper we will deal exclusively with the rank one case; i.e., flat complex line bundles. Henceforth, we simplify terminology and refer to $\rho$ as a line bundle.

Given a line bundle $\rho$, by choosing a non-zero vector $u_{i}$ in $V_{i}$ having unit norm, we can identify $V_{i}$ with $\mathbb{C} u_{i}$, the complex vector space spanned by $u_{i}$. Consequently, there is no loss in generality in assuming that $V_{i}=\mathbb{C}$ for every $i \in \Gamma_{0}$. In this instance, $\rho_{b}: \mathbb{C} \rightarrow \mathbb{C}$ is given by multiplication by a unit complex number, which by abuse of notation we denote as $\rho_{b}$. With respect to these choices, $\rho$ is given by a function $\Gamma_{1} \rightarrow U(1)$.

Recall that a circuit $C$ of $\Gamma$ is a simple closed path. An orientation of $C$ consists of a choice of direction for traversing $C$.

Definition 1.2. If $C$ is an oriented circuit of $\Gamma$, then the holonomy of $\rho$ along $C$ is given by the product

$$
\rho_{C}:=\prod_{b \in C_{1}} \rho_{b}^{s_{b}},
$$

where $s_{b}= \pm 1$ according as to whether the orientation of $C$ is the same as the orientation of $b$.

If $\bar{C}$ denotes $C$ with its reverse orientation, then $\rho_{\bar{C}}=\rho_{C}^{*}$, where $\rho_{C}^{*}$ denotes the complex conjugate of $\rho_{C}$.

More generally, suppose $A \subset \Gamma$ is a subgraph with the property that each component $A_{\alpha}$ of $A$ has trivial Euler characteristic. Then $A_{\alpha}$ has a unique circuit $C_{\alpha}$. Then $A$ has a preferred set of circuits. Assigning to $C_{\alpha}$ an arbitrary orientation, we set

$$
\hat{\rho}_{A}:=\prod_{\alpha}\left(\rho_{C_{\alpha}}-1\right)\left(\rho_{C_{\alpha}}^{*}-1\right)=\prod_{\alpha}\left(2-\rho_{C_{\alpha}}-\rho_{C_{\alpha}}^{*}\right),
$$

where $\alpha$ ranges over the components of $A$. This last expression is well-defined and independent of the choice of orientation for the circuits. It is also a real number.

\section{The twisted chain complex}

For $i=0,1$, let $C_{i}(\Gamma ; \rho)$ denote the $\mathbb{C}$-vector space having basis $\Gamma_{i}$. Define the twisted boundary operator

$$
\partial: C_{1}(\Gamma ; \rho) \rightarrow C_{0}(\Gamma ; \rho)
$$

by mapping an edge $b$ to the vector $\rho_{b} d_{0}(b)-d_{1}(b)$ and extending linearly. The homology of this two-stage complex is denoted by $H_{*}(\Gamma ; \rho)$. It is invariant with respect to barycentric subdivision. That is, if $\Gamma^{\prime}$ is the barycentric subdivision of $\Gamma$ and $\rho^{\prime}$ is a line bundle on $\Gamma^{\prime}$ such that $\rho_{b}=\rho_{b_{0}}^{\prime} \rho_{b_{1}}^{\prime}$ when $b=b_{0} b_{1}$ is the subdivision of an edge $b$, then $H_{*}(\Gamma ; \rho) \cong H_{*}\left(\Gamma^{\prime} ; \rho^{\prime}\right)$. Note that $H_{1}(\Gamma ; \rho)$ is a subspace of $C_{1}(\Gamma ; \rho)$ consisting of the cycles.

\footnotetext{
${ }^{1}$ Strictly speaking, what we have defined here is really the notion of a transport operator on $\Gamma$
} associated with a flat connection, but we will not need to worry about this distinction. 
Remark 1.3. This is the traditional notation. It is imprecise since each vector space $C_{i}(\Gamma ; \rho)$ does not depend on $\rho$ whereas the boundary operator $\partial$ does. A more precise notation would write the complex as $\partial_{\rho}: C_{1}(\Gamma ; \mathbb{C}) \rightarrow C_{0}(\Gamma ; \mathbb{C})$.

If $A \subset \Gamma$ is a subcomplex, we have the relative chain complex $C_{*}(\Gamma, A ; \rho)$ that is the quotient complex $C_{*}(\Gamma ; \rho) / C_{*}(A ; \rho)$. It has a basis consisting of the cells of $\Gamma$ that are not in $A$.

\section{The resistance operator}

A resistance function is a map $r: \Gamma_{1} \rightarrow \mathbb{R}_{+}$which assigns to an edge $b$ a resistance $r_{b}>0$. Associated with $r$ is the resistance operator

$$
R: C_{1}(\Gamma ; \rho) \rightarrow C_{1}(\Gamma ; \rho),
$$

which on basis elements is defined by $b \mapsto r_{b} b$.

\section{The standard and modified inner products}

The standard Hermitian inner product on $C_{1}(\Gamma ; \rho)$, denoted $\langle\cdot, \cdot \cdot$, is given on basis elements $b, b^{\prime} \in \Gamma_{1}$ by

$$
\left\langle b, b^{\prime}\right\rangle:=\delta_{b b^{\prime}},
$$

where $\delta_{b b^{\prime}}$ is Kronecker delta.

Associated with the resistance operator $R$ is the modified inner product on $C_{1}(\Gamma ; \rho)$, denoted $\langle\cdot, \cdot\rangle_{R}$, given by

$$
\left\langle b, b^{\prime}\right\rangle_{R}:=r_{b}\left\langle b, b^{\prime}\right\rangle=\delta_{b b^{\prime}} r_{b}
$$

\section{Twisted spanning trees}

From now on we assume that $\Gamma$ is connected and finite.

Definition 1.4. A $\rho$-spanning tree for $\Gamma$ is a subcomplex $T \subset \Gamma$ such that

- $T_{0}=\Gamma_{0}$,

- $H_{1}(T ; \rho)=0$, and

- The homomorphism $H_{0}(T ; \rho) \rightarrow H_{0}(\Gamma ; \rho)$ induced by the inclusion is an isomorphism.

Remark 1.5. When $\rho$ is the trivial line bundle, we recover the usual notion of spanning tree. In the next section we characterize the $\rho$-spanning trees of $\Gamma$.

We henceforth make the following assumption:

Assumption. The vector space $H_{0}(\Gamma ; \rho)$ is trivial.

Remark 1.6. The triviality of $H_{0}(\Gamma ; \rho)$ is equivalent to the statement that the holonomy over each cycle of $\Gamma$ is nontrivial. In the case of the twisted matrix-tree theorem (Theorem $\mathbb{Q}$ below), this assumption doesn't cause additional restrictions on generality: if $H_{0}(\Gamma ; \rho)$ is nontrivial, then the twisted Laplacian has trivial determinant.

Definition 1.7. The weight of a $\rho$-spanning tree $T$ is the real number

$$
w_{T}:=\hat{\rho}_{T} \prod_{b \in T_{1}} r_{b}^{-1}
$$


Remark 1.8. If we delete the factor $\hat{\rho}_{T}$ from the above expression, we obtain the weights appearing in classical untwisted version of the Kirchhoff formula (cf. [NS]).

\section{The operator $\bar{T}$}

Given a $\rho$-spanning tree $T$, we define an operator

$$
\bar{T}: C_{1}(\Gamma ; \rho) \rightarrow H_{1}(\Gamma ; \rho)
$$

as follows: if $b \in T_{1}$ then $\bar{T}(b)=0$. If $b \in \Gamma_{1} \backslash T_{1}$, we form the graph $T \cup b$. Then $\operatorname{dim}_{\mathbb{C}} H_{1}(T \cup b ; \rho)=1$. Let $c \in H_{1}(T \cup b ; \rho)$ be a non-zero vector, and set $t_{b}=\langle c, b\rangle$. We set $\bar{T}(b):=c / t_{b}$. This does not depend on the choice of $c$. Note that $H_{1}(T \cup b ; \rho) \rightarrow$ $H_{1}(\Gamma ; \rho)$ is an inclusion, so this definition makes sense.

Remark 1.9. It will be useful to have an alternative description of $\bar{T}$. Assume $b \in$ $\Gamma_{1} \backslash T_{1}$. The homology class $[\partial b] \in H_{0}(T ; \rho)=0$ is trivial, so $\partial b \in C_{0}(T ; \rho)$ bounds a chain $u \in C_{1}(T ; \rho)$. Then $c:=b-u \in C_{1}(T \cup b ; \rho)$ is a cycle such that $t_{b}=\langle c, b\rangle=1$. In this case $\bar{T}(b)=c$.

\section{The main results}

The twisted version of Kirchhoff's network theorem will be a consequence of having a concrete description of the projection operator from twisted 1-chains to twisted 1cycles.

Theorem A (Twisted Projection Formula). With respect to the modified inner product $\langle\cdot, \cdot\rangle_{R}$, the hermitian projection of $C_{1}(\Gamma ; \rho)$ onto the subspace $H_{1}(\Gamma ; \rho)$ is given by

$$
\frac{1}{\Delta} \sum_{T} w_{T} \bar{T}
$$

where $T$ ranges over the $\rho$-spanning trees of $\Gamma$ and $\Delta=\sum_{T} w_{T}$.

Our twisted version of Kirchhoff's network theorem is

Corollary $B$ (Twisted Network Theorem). Given a vector $\mathbf{V} \in C_{1}(\Gamma ; \rho)$, there is only one vector $z \in Z_{d}(\Gamma ; \rho)$ such that $\mathbf{V}-R z \in B^{1}(\Gamma ; \rho)$. Furthermore, for each edge $b$, we have

$$
\langle z, b\rangle=\frac{1}{\Delta} \sum_{T} \frac{w_{T}}{r_{b}}\langle\mathbf{V}, \bar{T}(b)\rangle .
$$

Remark 1.10. In the untwisted case $(\rho=1)$, this is the formulation of Kirchhoff's network theorem that is found in $[\mathbf{\mathbb { N } \mathbf { S }}]$. The expression $\langle\mathbf{V}, b\rangle$ is called the voltage source on the branch $b$ and $\langle z, b\rangle$ is the current residing on $b$.

Let

$$
\partial_{R}^{*}: C_{0}(\Gamma ; \rho) \rightarrow C_{1}(\Gamma ; \rho)
$$

be the formal adjoint to the boundary operator $\partial: C_{1}(\Gamma ; \rho) \rightarrow C_{0}(\Gamma ; \rho)$ with respect to the standard hermitian inner product on $C_{0}(\Gamma ; \rho)$ and the modified one on $C_{1}(\Gamma ; \rho)$, as determined by the resistance operator $R$.

The following is the result of Forman $[\mathbb{E}$, eq. (1)] that was alluded to above. 
Theorem $C$ (Twisted Weighted Matrix-Tree Theorem).

$$
\operatorname{det}\left(\partial \partial_{R}^{*}: C_{0}(\Gamma ; \rho) \rightarrow C_{0}(\Gamma ; \rho)\right)=\sum_{T} w_{T}
$$

where $T$ ranges over all $\rho$-spanning trees, and $w_{T}$ is as in Definition ㄸ. 7 .

The case $R=1$ is worth singling out. We use the notation $\partial^{*}=\partial_{R}^{*}$ in this case.

Corollary D (Twisted Matrix-Tree Theorem).

$$
\operatorname{det}\left(\partial \partial^{*}\right)=\sum_{T} \hat{\rho}_{T}
$$

where $T$ ranges over all $\rho$-spanning trees.

Remark 1.11. A natural question is whether versions of Theorems $\mathbf{Q}$ and $\mathbf{D}$ exist for higher rank bundles on $\Gamma$. We don't think this is likely, since our approach relies heavily on the fact that $U(1)$ is abelian.

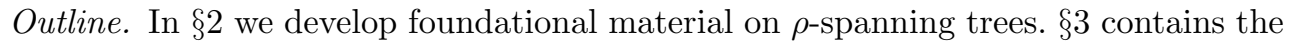

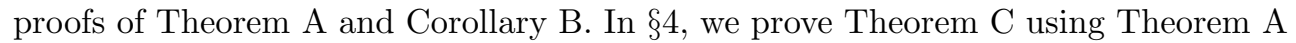
and the low temperature limit argument of [CCK].

\section{Acknowledgments}

The authors wish to Nikolai Sinitsyn for discussions related to the physical interpretation of the twisted network theorem. We are also indebted Misha Chertkov for hosting the first author and to Andrei Piryatinski for his unlimited hospitality. We thank the Los Alamos Center for Nonlinear Studies and the T-4 division for partially supporting this research. This material is based upon work supported by the National Science Foundation under Grant Nos. CHE-1111350 and DMS-1104355.

\section{Properties of twisted spanning trees}

The following lemma characterizes the structure of $\rho$-spanning trees of $\Gamma$. We remind the reader we have made the assumption throughout that $H_{0}(\Gamma ; \rho)=0$.

Lemma 2.1. A subcomplex $T \subset \Gamma$ is a $\rho$-spanning tree if and only if

- $T_{0}=\Gamma_{0}$,

- each connected component $T^{\alpha}$ of $T$ has trivial Euler characteristic; i.e., $T^{\alpha}$ possesses a unique circuit, $C_{\alpha}$ and

- the holonomy around $C_{\alpha}$ is nontrivial.

Remark 2.2. A cycle-rooted spanning forest (CRSF) is a subcomplex $T$ of $\Gamma$ satisfying the first two conditions listed in Lemma [.]; i.e., a $\rho$-spanning tree is a CRSF additionally satisfying the condition that the holonomy around circuits is nontrivial (cf. Fig. 四, [Kब, 4.1]). 


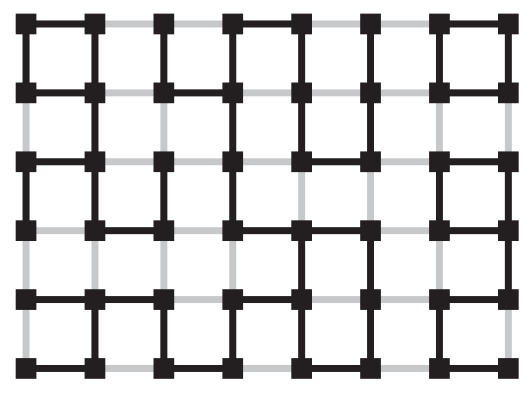

Figure 1: A lattice graph equipped with CRSF having four components (cf. Remark (2). The edges of the graph are the gray lines. The edges of the CRSF are indicated in black.

Proof of Lemma ש. Assume $T$ is a $\rho$-spanning tree. The assumption $H_{0}(\Gamma ; \rho)=$ 0 implies that $H_{0}(T ; \rho)=0$ and the latter implies $H_{0}\left(T^{\alpha} ; \rho\right)=0$ since $H_{0}(T ; \rho)=$ $\oplus_{\alpha} H_{0}\left(T_{\alpha} ; \rho\right)$. Similarly, $H_{1}(T ; \rho)=0$ implies $H_{1}\left(T^{\alpha} ; \rho\right)=0$. Hence the chain complex

$$
\partial: C_{1}\left(T^{\alpha} ; \rho\right) \rightarrow C_{0}\left(T^{\alpha} ; \rho\right)
$$

is acyclic. In particular, the number of edges of $T^{\alpha}$ equals the number of vertices, so the Euler characteristic of $T^{\alpha}$ is trivial. Orient the unique circuit $C_{\alpha}$ and let the holonomy around $C_{\alpha}$ be denoted $\rho_{\alpha}$. Then independence of twisted cohomology with respect to subdivision yields $H_{0}\left(T^{\alpha} ; \rho\right)=H_{0}\left(C_{\alpha} ; \rho\right)=H_{0}\left(S^{1} ; \rho_{\alpha}\right)$, where we are thinking of $S^{1}$ as a graph with one vertex and one edge and where the line bundle is given by $\rho_{\alpha}$. An easy calculation shows $H_{0}\left(S^{1} ; \rho_{C}\right)$ is the cokernel of the map $\left(\rho_{\alpha}-1\right): \mathbb{C} \rightarrow \mathbb{C}$. Hence the triviality of $H_{*}\left(T^{\alpha} ; \rho_{\alpha}\right)$ is equivalent to the statement $\rho_{\alpha} \neq 1$.

Conversely, given $T$ satisfying the three conditions, the second and third conditions imply $H_{*}(T ; \rho)=\oplus_{\alpha} H_{*}\left(T^{\alpha} ; \rho\right)$ is trivial. Hence $T$ is a $\rho$-spanning tree.

Lemma 2.3. $\Gamma$ has a $\rho$-spanning tree.

Proof. Call an edge $b$ of $\Gamma$ essential if there is a cycle $z \in H_{1}(\Gamma ; \rho) \subset C_{1}(\Gamma ; \rho)$ such that $\langle b, z\rangle \neq 0$. If there is no such edge, then it is straightforward to check that $\Gamma$ is a $\rho$-spanning tree.

Assume then that there is an essential edge $b$. Let $Y$ be the effect of removing (the interior of) $b$ from $\Gamma$. Then we have a short exact sequence

$$
0 \rightarrow H_{1}(Y ; \rho) \rightarrow H_{1}(\Gamma ; \rho) \rightarrow H_{1}(b, \partial b ; \rho) \rightarrow H_{0}(Y ; \rho) \rightarrow 0
$$

and the condition $\langle b, z\rangle \neq 0$ implies that the homomorphism $H_{1}(\Gamma ; \rho) \rightarrow H_{1}(b, \partial b ; \rho)$ is nontrivial (note that $H_{1}(b, \partial b ; \rho) \cong \mathbb{C}$ ). It follows that $\operatorname{dim}_{\mathbb{C}} H_{1}(Y ; \rho)<\operatorname{dim}_{\mathbb{C}} H_{1}(\Gamma ; \rho)$ and $H_{0}(Y ; \rho)=0$. We do not require that $Y$ be connected. We now replace $\Gamma$ by $Y$ and iterate this construction until we obtain a subcomplex $T$ having no essential cells and $H_{*}(T ; \rho)=0$. Then $T$ is a $\rho$-spanning tree.

Lemma 2.4. Fix a $\rho$-spanning tree $T$ and let $b_{1}, \ldots, b_{k}$ be the set of edges of $\Gamma_{1} \backslash T_{1}$. Then $\left\{\bar{T}\left(b_{1}\right), \ldots, \bar{T}\left(b_{k}\right)\right\}$ is a basis for $H_{1}(\Gamma ; \rho)$. 
Proof. The homomorphism $H_{1}(\Gamma ; \rho) \rightarrow H_{1}(\Gamma, T ; \rho)$ is an isomorphism. Furthermore, $H_{1}(\Gamma, T ; \rho)=C_{1}(\Gamma ; T ; \rho)$ has basis $\left\{b_{1}, \ldots, b_{k}\right\}$. The inverse homomorphism sends $b_{i}$ to $\bar{T}\left(b_{i}\right)$.

Corollary 2.5. For any $z \in H_{1}(\Gamma ; \rho)$, we have $\bar{T}(z)=z$.

Proof. The definition of $\bar{T}$ shows $\bar{T}^{2}\left(b_{i}\right)=\bar{T}\left(b_{i}\right)$. Write $z=\sum_{i} a_{i} \bar{T}\left(b_{i}\right)$. Then

$$
\bar{T}(z)=\sum_{i} a_{i} \bar{T}^{2}\left(b_{i}\right)=\sum_{i} a_{i} \bar{T}\left(b_{i}\right)=z
$$

Given a $\rho$-spanning tree $T$, consider an edge $b_{i} \in \Gamma_{1} \backslash T_{1}$ as well as an edge $b_{j} \in T_{1}$. Let $U=\left(T \backslash b_{j}\right) \cup b_{i}$.

Lemma 2.6. $U$ is a $\rho$-spanning tree if and only if $\left\langle\bar{T}\left(b_{i}\right), b_{j}\right\rangle \neq 0$.

Proof. Throughout this proof we use local coefficients in $\rho$ but suppress this from the notation. We have an exact sequence

$$
0 \rightarrow H_{1}\left(T \backslash b_{j}\right) \rightarrow H_{1}(T) \rightarrow H_{1}\left(b_{j}, \partial b_{j}\right) \rightarrow H_{0}\left(T \backslash b_{j}\right) \rightarrow 0,
$$

where we are using the fact that $H_{0}(T)=0$. Since $H_{1}(T)=0$ and $\operatorname{dim}_{\mathbb{C}} H_{1}\left(b_{j}, \partial b_{j}\right)=$ 1, we infer that $H_{1}\left(T \backslash b_{j}\right)=0$ and $\operatorname{dim}_{\mathbb{C}} H_{0}\left(T \backslash b_{j}\right)=1$.

The inclusion $U \subset T \cup b_{i}$ induces another exact sequence

$$
0 \rightarrow H_{1}(U) \rightarrow H_{1}\left(T \cup b_{i}\right) \rightarrow H_{1}\left(b_{j}, \partial b_{j}\right) \rightarrow H_{0}(U) \rightarrow 0,
$$

and the homomorphism $H_{1}\left(T \cup b_{i}\right) \rightarrow H_{1}\left(b_{j}, \partial b_{j}\right)$ is a map of rank one vector spaces that is induced by sending the preferred cycle $c \in H_{1}(T \cup b)$ to $\left\langle\bar{T}\left(b_{i}\right), b_{j}\right\rangle$ with respect to the preferred identification $H_{1}(b, \partial b) \cong \mathbb{C}$. Consequently, $U$ is a $\rho$-spanning tree if and only if $\left\langle\bar{T}\left(b_{i}\right), b_{j}\right\rangle \neq 0$.

Proposition 2.7. With $b_{i}, b_{j}, T, U$ as above, we have

$$
\hat{\rho}_{T}\left\langle\bar{T}\left(b_{i}\right), b_{j}\right\rangle=\hat{\rho}_{U}\left\langle b_{i}, \bar{U}\left(b_{j}\right)\right\rangle .
$$

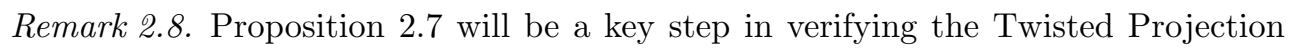
Formula (Theorem $\mathbb{Q}$ ). Although we will have managed to reduce most of the argument to algebraic topology, we cannot completely eliminate combinatorics from the

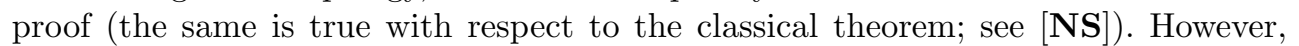
Proposition $\square]$ effectively minimizes the role of combinatorics to a kind of general and relatively simple statement.

Proof of Proposition [.7. There are two cases to consider: either $b_{i}$ is attached to two distinct components of $T$ or $b_{1}$ is attached to a single component of $T$. We proceed by direct calculation in either case. Figure $\square$ gives a visualization of the cases at hand.

Case 1: Assume that $b_{i}$ is attached to two distinct components of $T$, say $A$ and $B$. By switching the roles of $A$ and $B$ if necessary, we may suppose that $\left(d_{0}\left(b_{i}\right), d_{1}\left(b_{i}\right)\right)=$ $(w, v)$, where $v$ lies in $A$ and $w$ lies in $B$. Without loss of generality assume that $b_{j}$ lies in $A$. Let $C$ be the unique circuit of $A$ and $C^{\prime}$ the unique circuit of $B$. 
Then

$$
v=\partial c
$$

for $c \in C_{1}(A ; \rho)$. We may then write $c=c_{0}+\alpha b_{j}$, where $\alpha \in \mathbb{C}$ and $\left\langle c_{0}, b_{j}\right\rangle=0$. Similarly, we write $w=\partial d$, where $d \in C_{1}(B ; \rho)$. Then $\left\langle\bar{T}\left(b_{i}\right), b_{j}\right\rangle$ equals

$$
\left\langle b_{i}-\left(\rho_{b_{i}} d-c_{0}-\alpha b_{j}\right), b_{j}\right\rangle=\alpha,
$$

since $\partial\left(\rho_{b_{i}} d-c_{0}-\alpha b_{j}\right)=\partial b_{i}$ and $\rho_{b_{i}} d-c_{0}-\alpha b_{j}$ is a chain of $T$.

A similar calculation shows $\left\langle b_{i}, \bar{U}\left(b_{j}\right)\right\rangle$ equals

$$
\left\langle b_{i}, b_{j}-\frac{\rho_{b_{i}} d-b_{i}-c_{0}}{\alpha}\right\rangle=\left(\alpha^{-1}\right)^{*} .
$$

In order to compute $\alpha$, it is enough to identify the 1 -chain $c \in C_{1}(A ; \rho)$ whose boundary equals $v$, since then $\left\langle c, b_{j}\right\rangle=-\alpha$.

To find $c$ we rename $v=v_{1}$ and choose a vertex $v_{k}$ on the unique circuit of $A$ together with an embedded path of edges $e_{1}, \ldots e_{k}$ that connects $v_{1}$ to $v_{k}$. Without loss of generality, we can assume that none of the edges $e_{i}$ lies in the unique cycle of $A$. Let $e_{k+1}, \ldots, e_{n}$ denote the sequence of edges given by the traversing the unique cycle of $A$ such that $v_{k}$ is a vertex of both $e_{k+1}$ and $e_{n}$. Then $c$ is a linear combination of the edges $e_{i}$, which can be explicitly computed using the fact that $\partial e_{i}=\rho_{e_{i}} d_{0}\left(e_{i}\right)-d_{1}\left(e_{i}\right)$. Then a straightforward calculation yields the expression for the component of $c$ along the edge $e_{i}$ as

$$
\left\langle c, e_{i}\right\rangle=-\frac{\rho_{1}^{s_{1}} \ldots \rho_{i-1}^{s_{i-1}}}{\rho_{A}-1},
$$

where $\rho_{i}:=\rho_{e_{i}}$ and $s_{i}= \pm 1$ according as to whether $e_{i}$ points in the direction of the path or not (we have also oriented $A$ in a way that is compatible with our choice of path). In particular, $b_{j}=e_{\ell}$ for some index $\ell$, so

$$
\alpha=\frac{\rho_{1}^{s_{1}} \ldots \rho_{\ell-1}^{s_{\ell-1}}}{\rho_{A}-1} .
$$

Since $\hat{\rho}_{T}=\hat{\rho}_{A} \hat{\rho}_{B} \hat{\rho}^{\prime}$, where $\hat{\rho}^{\prime}$ is the product of the $\hat{\rho}_{T^{\alpha}}$ ranging over the remaining components of $T$, we have

$$
\hat{\rho}_{T} \alpha=\rho_{1}^{s_{1}} \ldots \rho_{i-1}^{s_{\ell-1}}\left(\rho_{A}^{*}-1\right) \hat{\rho}_{B} \hat{\rho}^{\prime}
$$

Since $\hat{\rho}_{U}=\hat{\rho}_{B} \hat{\rho}^{\prime}$, we see

$$
\hat{\rho}_{U}\left(\alpha^{-1}\right)^{*}=\hat{\rho}_{T} \alpha,
$$

which concludes Case 1.

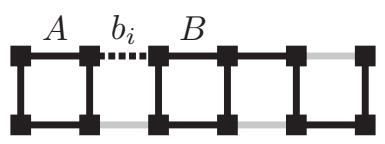

(a) $b_{i}$ bridges two distinct components of $T$

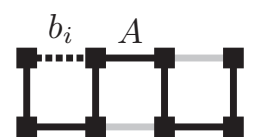

(b) $b_{i}$ attached to a single component of $T$

Figure 2: The two cases of Proposition [2.7 
Case 2: In this instance $b_{i}$ is attached to a single component $A$ of $T$. In this case we need to find a 1-chain $c$ of $A$ such that $\partial c=\partial b_{i}$. Arguing in a way analogous to the beginning of Case 1 , if we set

$$
\left\langle\bar{T}\left(b_{i}\right), b_{j}\right\rangle=\alpha
$$

then it follows that

$$
\left\langle\bar{b}_{i}, \bar{U}\left(b_{j}\right)\right\rangle=\left(\alpha^{-1}\right)^{*} .
$$

Suppose $d_{1}\left(b_{i}\right)=v$ and $d_{0}\left(b_{i}\right)=w$ (where it is possible that $v=w$ ). We select a simple path $e_{1}, \ldots, e_{k}$ of edges of $A$ such that $v$ meets $e_{1}$ and $w$ meets $e_{k}$. Let us rename $b_{i}$ as $e_{k+1}$. Then $c$ is a linear combination of the edges $e_{i}$ for $1 \leqslant i \leqslant k$. Let $C$ denote the unique circuit of $A$. Then $A$ decomposes as

$$
A_{-} \cup C \cup A_{+}
$$

in which $A_{-}$is a connected subgraph of $A$ that meets the vertex $v$ and $A_{+}$is the connected subgraph of $A$ that meets the vertex $w$. For a given index $i$, consider the expressions

$$
\alpha_{-}:=\prod_{1 \leqslant j<i} \rho_{j}^{s_{j}} \quad \alpha_{+}:=\prod_{i \leqslant j \leqslant k+1} \rho_{j}^{s_{j}} .
$$

A calculation similar to that appearing in Case 1 gives, for $0 \leqslant i \leqslant k$,

$$
\left\langle c, e_{i}\right\rangle= \begin{cases}\alpha_{-} & \text {if } e_{i} \subset A_{-}, \\ \left(\rho_{A}-1\right)^{-1}\left(\alpha_{+}-\alpha_{-}\right) & \text {if } e_{i} \subset C, \\ \alpha_{+} & \text {if } e_{i} \subset A_{+} .\end{cases}
$$

Then if $\beta_{j}=e_{\ell}$ for some $\ell$, we have $\left\langle\bar{T}\left(\beta_{i}\right), \beta_{j}\right\rangle=-\left\langle c, e_{\ell}\right\rangle$. As before, we have $\hat{\rho}_{T}=$ $\hat{\rho}_{A} \hat{\rho}^{\prime}$, where $\hat{\rho}^{\prime}$ is a product of $\hat{\rho}_{T^{\alpha}}$ for $T^{\alpha}$ ranging over the other components of $T$. Consequently,

$$
\left\langle\hat{\rho}_{T} \bar{T}\left(\beta_{i}\right), \beta_{j}\right\rangle=\hat{\rho}_{A} \hat{\rho}^{\prime} \alpha,
$$

where $\alpha=-\left\langle c, e_{\ell}\right\rangle$ is explicitly given by Eq. (G).

The remainder of the argument is just as in Case 1. A straightforward calculation that we omit shows

$$
\hat{\rho}_{U}= \begin{cases}\hat{\rho}_{T} & \text { if } b_{j} \subset A_{-} \cup A_{+}, \\ \left(2+\alpha_{+} \alpha_{-}^{*}-\alpha_{+}^{*} \alpha_{-}\right) \hat{\rho}^{\prime} & \text { if } b_{j} \subset C .\end{cases}
$$

Then use Eq. (回) and Eq. (四) to identify the product $\hat{\rho}_{U}\left(\alpha^{-1}\right)^{*}$. We infer that it coincides with $\hat{\rho}_{T} \alpha$, thereby completing the proof.

\section{Proof of Theorem $\mathbb{A}$ and Corollary $\mathbb{B}$}

Lemma 3.1. For distinct edges $b_{i}, b_{j} \in \Gamma_{1}$, let $\mathcal{T}_{i j}$ be the set of $\rho$-spanning trees such that $\left\langle\bar{T}\left(b_{i}\right), b_{j}\right\rangle \neq 0$. Then

$$
\sum_{T \in \mathcal{T}_{i j}} w_{T}\left\langle\bar{T}\left(b_{i}\right), b_{j}\right\rangle_{R}=\sum_{U \in \mathcal{T}_{j i}} w_{U}\left\langle b_{i}, \bar{U}\left(b_{j}\right)\right\rangle_{R}
$$


Proof. From the definition of the weights, we have

$$
\frac{r_{j} w_{T}}{\hat{\rho}_{T}}=\frac{r_{i} w_{U}}{\hat{\rho}_{U}}
$$

Recall that $\left\langle\bar{T}\left(b_{i}\right), b_{j}\right\rangle_{R}=r_{j}\left\langle\bar{T}\left(b_{i}\right), b_{j}\right\rangle$. Using Eq. (区) and Proposition [2.], we infer

$$
w_{T}\left\langle\bar{T}\left(b_{i}\right), b_{j}\right\rangle_{R}=w_{U}\left\langle b_{i}, \bar{U}\left(b_{j}\right)\right\rangle_{R} .
$$

Now sum up over all $T \in \mathcal{T}_{i j}$.

Proof of Theorem $\mathbf{⿴ 囗 十}$. Consider the operator $F:=\sum_{T} w_{T} \bar{T}$, where the sum is over all $\rho$-spanning trees of $\Gamma$. For any pair of edges $b_{i}$ and $b_{j}$ of $\Gamma$ we have

$$
\begin{aligned}
\left\langle\sum_{T} w_{T} \bar{T}\left(b_{i}\right), b_{j}\right\rangle_{R} & =\sum_{T \in \mathcal{T}_{i j}} w_{T}\left\langle\bar{T}\left(b_{i}\right), b_{j}\right\rangle_{R} \\
& =\sum_{U \in \mathcal{T}_{j i}} w_{U}\left\langle b_{i}, \bar{U}\left(b_{j}\right)\right\rangle_{R} \quad \text { by Lemma } \boldsymbol{C . T}, \\
& =\left\langle b_{i}, \sum_{U} w_{U} \bar{U}\left(b_{j}\right)\right\rangle_{R} \\
& =\left\langle b_{i}, \sum_{T} w_{T} \bar{T}\left(b_{j}\right)\right\rangle_{R}
\end{aligned}
$$

Hence $F$ is self-adjoint in the modified inner product.

If $z \in Z_{1}(\Gamma ; \rho)$, then using Corollary 2.5 , we have

$$
F(z)=\left(\sum_{T} w_{T}\right) \bar{T}(z)=\left(\sum_{T} w_{T}\right) z=: \Delta z
$$

Consequently, $(1 / \Delta) F$ restricts to the identity on $Z_{d}(X ; \rho)$. As $(1 / \Delta) F$ is self-adjoint, it is the Hermitian projection in the modified inner product.

Proof of Corollary $\mathbb{B}$. Let $z$ be the Hermitian projection of $R^{-1} \mathbf{V}$ in the modified inner product. Then $R^{-1} \mathbf{V}-z \in B_{R}^{d}(\Gamma ; \rho)$; i.e.,

$$
0=\left\langle R^{-1} \mathbf{V}-z, z^{\prime}\right\rangle_{R}=\left\langle\mathbf{V}-R z, z^{\prime}\right\rangle
$$

for all $z^{\prime} \in Z_{d}(X ; \rho)$. Hence, $\mathbf{V}-R z \in B^{d}(\Gamma ; \rho)$. The uniqueness of $z$ is a consequence of the fact that $B^{d}(\Gamma ; \rho)$ is the orthogonal complement to $Z_{d}(X ; \rho)$ in the standard inner product.

The proof of the last part is given by direct calculation using the self-adjointness of the operator $\sum_{T} w_{T} \bar{T}$ :

$$
\begin{aligned}
\langle z, b\rangle & =\frac{1}{r_{b}}\langle z, b\rangle_{R}, \\
& =\frac{1}{r_{b}}\left\langle\frac{1}{\Delta} \sum_{T} w_{T} R^{-1} \mathbf{V}, b\right\rangle_{R}, \\
& =\frac{1}{\Delta} \sum_{T} \frac{w_{T}}{r_{b}}\left\langle R^{-1} \mathbf{V}, \bar{T}(b)\right\rangle_{R}, \\
& =\frac{1}{\Delta} \sum_{T} \frac{w_{T}}{r_{b}}\langle\mathbf{V}, \bar{T}(b)\rangle .
\end{aligned}
$$




\section{Proof of Theorem $\mathbb{C}$}

The proof of Theorem $\mathbb{Q}$ is essentially the same as the proof of [CCK, theorem C]. We will outline the essential steps. The first step is to show that

$$
\operatorname{det}\left(\partial \partial_{R}^{*}: C_{0}(\Gamma ; \rho) \rightarrow C_{0}(\Gamma ; \rho)\right)=\gamma \sum_{T} w_{T},
$$

where $T$ ranges over all $\rho$-spanning trees, and the pre-factor $\gamma$ is to be determined. This step follows, mutatis mutandis, by the proof of [CCK], proposition 4.2]. We emphasize that $\gamma$ is independent of $R$.

The second and final step is to compute the prefactor $\gamma$ and show that it equals 1 . We work perturbatively, following a modified version of [CCK, proposition 5.2]. To this end, let $\beta \in \mathbb{R}_{+}$be the perturbation parameter and fix a $\rho$-spanning tree $T$. For any $W: \Gamma_{1} \rightarrow \mathbb{R}$, write $R=e^{W}, R_{\beta}=e^{\beta W}$, and set $\mathcal{L}_{R}=\partial \partial_{R}^{*}$. Define $\mathcal{L}_{R}^{T}=$ $\partial_{T} e^{-W} \partial_{T}^{*}: C_{0}(T ; \rho) \rightarrow C_{0}(T ; \rho)$.

A choice of orthogonal projection $C_{1}(\Gamma ; \rho) \rightarrow C_{1}(T ; \rho)$ allows us to write

$$
\mathcal{L}_{R}=\mathcal{L}_{R}^{T}+\delta \mathcal{L} .
$$

A standard expansion of the above operator allows us to bound the elements of $\delta \mathcal{L}$

$$
\left|\delta \mathcal{L}_{j k}\right| \leqslant e^{-\beta \min _{b} \in \Gamma_{1} \backslash T_{1} W_{b}} B,
$$

where $B$ is independent of $W$ and $\beta$. Since $\gamma$ is independent of $R$, we choose $W: \Gamma_{1} \rightarrow$ $\mathbb{R}$ so that

$$
W_{b}>\sum_{\alpha \in T_{1}} W_{\alpha}-k \min _{b^{\prime} \in T_{1}} W_{b^{\prime}} \quad \text { for any } b \in \Gamma_{1} \backslash T_{1},
$$

where $k$ is number of edges of $\Gamma$. Our choice of $W$ implies that in the $\beta \rightarrow \infty$ limit, the terms arising from $\mathcal{L}_{R}^{T}$ dominate those of $\delta \mathcal{L}$. Therefore,

$$
\lim _{\beta \rightarrow \infty} \frac{\operatorname{det} \mathcal{L}_{R_{\beta}}^{T}}{\operatorname{det} \mathcal{L}_{R_{\beta}}}=1
$$

Substituting $\beta W$ for $W$ in Eq. (四), taking the $\beta \rightarrow \infty$ limit, substituting the relation (四), and doing some minor rewriting, we deduce

$$
\operatorname{det}\left(\mathcal{L}_{R}^{T}\right)=\gamma w_{T}
$$

Note that $\mathcal{L}_{R}^{T}=\partial_{T} e^{-W} \partial_{T}^{*}$, and by definition of $w_{T}$, we have $\operatorname{det} e^{-W}=\hat{\rho}_{T}^{-1} w_{T}$. Consequently,

$$
\operatorname{det}\left(\mathcal{L}_{R}^{T}\right)=\hat{\rho}_{T}^{-1} w_{T} \operatorname{det}\left(\partial_{T} \partial_{T}^{*}\right)
$$

It follows that

$$
\gamma=\hat{\rho}_{T}^{-1} \operatorname{det}\left(\partial_{T} \partial_{T}^{*}\right)
$$

Theorem $\mathbb{Q}$ is then a consequence of the following.

Lemma 4.1. For any $\rho$-spanning tree $T$, we have

$$
\operatorname{det}\left(\partial_{T} \partial_{T}^{*}\right)=\hat{\rho}_{T} .
$$

Hence, $\gamma=1$. 
Proof. Clearly both sides of the equation factor as a product over the connected components of $T$. So if $T^{\alpha}$ is a component of $T$, it will suffice to show

$$
\operatorname{det}\left(\partial_{T^{\alpha}} \partial_{T^{\alpha}}^{*}\right)=\hat{\rho}_{T^{\alpha}} .
$$

This last statement can be proved in a number of ways. For example, Kenyon [Ke] proves it using an interpretation of the determinant as a summation of cycles over the symmetric group. We will give a proof using gauge invariance.

The gauge group $G$ of $\Gamma$ is the group of functions $\Gamma_{0} \rightarrow U(1)$ with respect to pointwise multiplication. It is convenient in what follows to set $g_{v}=g(v)$ for a vertex $v$. Then $G$ acts on line bundles according to the rule

$$
g \cdot \rho(b)=g_{d_{0}(b)} g_{d_{1}(b)}^{*} \rho_{b} .
$$

Set $\rho^{g}=g \cdot \rho$. To distinguish between boundary operators, we write $\partial$ for the boundary operator associated with $\rho$, and $\partial_{g}$ for the one associated with $\rho^{g}$. Define an action

$$
G \times C_{0}(\Gamma ; \rho) \rightarrow C_{0}(\Gamma ; \rho)
$$

by $g \cdot v=g_{v} v$, for $v \in \Gamma_{0}$.

It is then straightforward to check that for $g \in G$ we have

$$
\partial_{g} \partial_{g}^{*}=g \partial \partial^{*} g^{-1} \text {. }
$$

In particular, $\operatorname{det}\left(\partial_{g} \partial_{g}^{*}\right)=\operatorname{det}\left(\partial \partial^{*}\right)$.

Write $T^{\alpha}=A \cup b$, where $A$ is tree in the classical sense. We claim that there is a gauge $g \in G$ such that $\partial_{g}(e)=1$ for $e \in A_{1}$. To find $g$ we need to know that the system of equations

$$
g_{d_{0}(e)} g_{d_{1}(e)}^{*} \rho_{e}=1, \quad e \in A
$$

admits a solution. If we fix a vertex $i \in A_{0}$, we can set $g_{i}=1$. Then for any edge $i j$ of $A$ that connects $i$ to $j$, we set $g_{j}=\rho_{i j}^{s}$ with $s= \pm 1$ according to whether $i j$ points inward toward $i$ or not. Consider a vertex $k \neq i$ such that $j k$ is an edge of $A$. We set $g_{k}=\rho_{j k}^{s} g_{j}$, where in this instance $s$ is \pm 1 according to whether the edge $j k$ points toward $j$ or not. Continuing in this fashion, we obtain a solution to the system (四). For any vertex $i$ not in $A$ we set $g_{i}=1$. With respect to our choice of $g$, inspection shows that $\rho_{b}^{g}$ is the holonomy with respect to $\rho$ around the unique circuit of $A \cup b$ that is oriented in the direction of $b$. Hence, we can without loss of generality assume that the original line bundle $\rho$ is trivial on every edge other than $b$, and we are reduced to proving that $\operatorname{det}\left(\partial \partial^{*}\right)=\left(\rho_{b}-1\right)\left(\rho_{b}^{*}-1\right)$, where $\partial$ is the boundary operator for $T^{\alpha}=A \cup b$.

The columns of the matrix associated with $\partial$ represent the edges of $A \cup b$ and the rows represent the vertices. An edge $e$ of $A \cup b$ is said to be loose if it is attached to a vertex $i$ such that no other edge of $A \cup b$ is attached to $i$. If $e$ is loose, then the $e$-th column of $\partial$ has exactly two non-zero entries that are \pm 1 and these are of opposite sign. We infer that the determinant of $\partial$ remains unchanged when we remove the edge $e$ and the vertex $i$ from $A \cup b$. Iterating this procedure, we may assume without loss of generality that $A \cup b$ has no free edges. This means $A \cup b$ is a circuit. The determinant of $\partial$ in this case is easy to compute and is given by $\pm\left(\rho_{b}-1\right)$. Hence the determinant of $\partial^{*} \partial$ is $\left(\rho_{b}-1\right)\left(\rho_{b}^{*}-1\right)$. 


\section{References}

[B] Bollobás, B., Modern graph theory, Graduate Texts in Mathematics, 184, Springer-Verlag, New York, 1998.

[CCK] Catanzaro, M.J., Chernyak, V.Y., and Klein, J.R., Kirchhoff's theorems in higher dimensions and Reidemeister torsion, arXiv preprint 1206.6783.

[CKS1] Chernyak, V.Y., Klein, J.R., and Sinitsyn, N.A., Quantization and Fractional Quantization of Currents in Periodically Driven Stochastic Systems I: Average Currents, J. Chem. Phys. 136, 154107 (2012).

[CKS2] Chernyak, V.Y., Klein, J.R., and Sinitsyn, N.A., Quantization and Fractional Quantization of Currents in Periodically Driven Stochastic Systems II: Full Counting Statistics, J. Chem. Phys. 136, 154108 (2012).

[E] Eckmann, B., Harmonische Funktionen und Randwertaufgaben in einem Komplex. Comment. Math. Helv. 17 (1945), 240-255.

[F] Forman, R., Determinants of Laplacians on graphs, Topology 32 (1993), $35-46$.

[Ke] Kenyon, R., Spanning forests and the vector bundle Laplacian, Ann. Probab. 39 (2011), 1983-2017.

[NS] Nerode, A. and Shank H., An algebraic proof of Kirchhoff's network theorem, Amer. Math. Monthly 68 (1961), 244-247.

[R] Roth, J.P., An application of algebraic topology to numerical analysis: on the existence of a solution to the network problem, Proc. Nat. Acad. Sci. U.S.A. 41 (1955), 518-521.

Michael J. Catanzaro mike@math.wayne.edu

Department of Mathematics, Wayne State University, Detroit, MI 48202, USA

Vladimir Y. Chernyak chernyak@chem.wayne.edu

Department of Chemistry, Wayne State University, Detroit, MI 48202, USA

John R. Klein Eleinomath.wayne.edu

Department of Mathematics, Wayne State University, Detroit, MI 48202, USA 